\title{
Challenges for Emirati university students in comprehending mathematical text and word problems
}

\author{
Mark Causapin ${ }^{1}$, Timothy Groombridge2, * \\ ${ }^{1}$ Mathematics and Statistics, Zayed University, Abu Dhabi, United Arab Emirates \\ 2,* English and Writing, Zayed University, Abu Dhabi, United Arab Emirates \\ For correspondence: mark.causapin@zu.ac.ae, timothy.groombridge@zu.ac.ae
}

\begin{abstract}
The United Arab Emirates has been rapidly developing its educational institutions and trying to adopt a western system of higher education. The government has chosen English to be the medium of instruction in universities and thus has become a critical factor in student learning. In mathematics classes, it can be seen that students struggle to grasp concepts not just because of the actual content but also because of the language barrier. Language is intertwined with the multiple facets of mathematics education but one of the more salient areas is in comprehending mathematical text and problems. This paper attempts to summarize previous research findings that are related to this issue and describe problems faced by teachers using actual classroom cases. The conclusion of this paper outlines some tentative recommendations and a future research agenda to develop strategies to resolve these issues.
\end{abstract}

Keywords: Mathematics Education, Word Problems, Arabic, United Arab Emirates, English Language Learners

\section{Introduction}

During an exam, a student asked, "When do we use discounts in this word problem?" The word was not "discount" but "biscuit." For him, these two words looked very similar, with the $b$ and $d$ in the beginning, and the $s, c$, and $t$ all in the same position. Another student says, "Mister, this test is very different from the review questions we had!" It was in fact the same except for the contextualization of the word problems, yet it seemed very foreign for the students. In this situation, it was clear that many of them did not fully understand the meaning of the questions they had been presented.

In other instances like this, students were using surface clues they had instead of comprehending the word problems. For example, if a question that was solved in class had three embedded numbers and they were used in a particular order, the students might then reproduce the same process in the assessments without understanding the logical succession of problem solving procedures. Similar to what other researchers have found, they may start looking for key words like mean, greater than, or standard deviation and pick out the numbers beside them that they can use for a formula (De Corte et al., 1985). In one instance, a student was confused with the phrase, "a simple randomly selected sample of 50" because he was used to finding a number after "sample" and he unexpectedly saw the word "simple" which looked the same. Normally, the circumventing techniques he had developed worked but in this case it did not. Students might have been relying on the structure of the assessment questions rather than truly understanding the word problems. Morales (1998) was able to document this in a cohort of Mexican-American elementary students and psycholinguists had found the same (as cited in Clements, 1986; Siegel, 1978) (Adetula, 1990). It was likely that these "pseudo-analytic thought processes" (Vinner, 1997) made them successful throughout their student lives, but they might also have missed many important ideas and concepts because of difficulties with the English language.

Many students, not just at Zayed University (ZU) but also in the whole of the United Arab Emirates (UAE), encounter such linguistic difficulties. The country is relatively new and its first university was founded in 1977 (Kirk et al., 2010). ZU was established in 1998. Since the formation of these universities, a western model of higher education with English as the primary language has been 
adopted. At $\mathrm{ZU}$, incoming students are required to have an average score of at least 5 in the International English Language Testing System (IELTS). This test has a scoring scale from 1 to 9 , with 1 representing non-users and 9, expert users of English (IELTS, 2014). Listening, reading, writing, and speaking are assessed, and the overall average may reach 5 despite students obtaining low reading or writing scores. A score of 5 corresponds to modest users - those with a partial command of the language and who can handle basic communication in one's own field (IELTS, 2014). In 2008, around $85 \%$ attended preparatory English classes collectively called the Academic Bridge Program (ABP) to get them ready to attend their regular university classes (ZU, 2008). At that time, the required entry score was 5.5. The most problematic area for the students was reading, which led the university to incorporate more of this key skill area in the first and second year writing classes (ZU, 2012b). Available data shows that in 2011, students exiting the ABP had an average reading score of 4.8, and in Fall 2010, the reading score for students in their majors ( $3^{\text {rd }}$ and $4^{\text {th }}$ years) was 5.7 (ZU, 2012a). Given these relatively low scores, it is not surprising that mathematics learning is also affected.

The level of language skills the students have affects their mathematical development. Mathematics is not a universal language (Gorgorió et al., 2001) and it is incorrect to believe that it is free from linguistic issues (Austin et al., 1979; Barwell et al., 2007; Clarkson, 1983; Janzen, 2008; Ríordáin et al., 2009; Verzosa et al., 2013; Zepp, 1981). It is hypothesized that the relationship of English reading skills and mathematics performance might not even be linear - that there is a minimum reading level associated with improved performance (Beal et al., 2010). These issues present themselves in class not only in reading but also in other areas simultaneously: when instructors deliver lectures; when students listen to the instructor; during discourse between students and instructors and among students; when reading textbooks; during note-taking; when understanding questions in exercises and exams; and so on. Instructors of English Language Learners (ELL) have multiple issues to tackle such as resolving issues on how to deliver lessons, how to write questions and notes, how to contextualize problems and engage students in discussion. Accordingly, linguistic theories and meta-theories have been formulated covering the whole area of language and mathematics that serve as frameworks for understanding these issues such as the "mathematics is a language" metaphor (Pimm, 1987), mathematical registers (Halliday, 1978), "mathematics as multi-semiotic" (Lemke, 2003; O'Halloran, 1998), and the different views of language in research on language and mathematics (Solano-Flores, 2010).

In this paper, the focus is on written mathematical text from assessments and handouts that students need to understand and interpret. Although there are other areas to analyze, in practice this is a good starting point because of the students' weakness in reading. Understanding the underlying mathematical problem structure embedded in the text is the most basic difficulty students have (Bernardo, 1999). A. M. Ferguson et al. (1985) argue that these texts are more difficult to understand because they are very dense conceptually compared to regular prose; the writing style is different; they lack the rich context clues; words with familiar meanings may have different mathematical meanings; the continuity found in prose - the subjects and ideas from one sentence or paragraph to another - is absent in mathematical word problems; and the reading patterns necessary for prose are not applicable (as cited from Vos, 1979). In addition to being conceptually dense, texts are interpersonally alienating, highly structured textually in unfamiliar ways, and multi-semiotic - involving natural language, symbolism, and visuals such as graphs, charts, and diagrams (Schleppegrell, 2010).

It is not being proposed that English be abandoned as a medium of instruction for mathematics. Arguing for or against this language policy is a political question and goes beyond the scope of this paper (Gutstein, 2007). Furthermore, given the characteristics of mathematical text, students would still have difficulties even if they used Arabic. The purpose of this study is to demonstrate that there are language issues affecting mathematics learning that need to be resolved and to suggest a direction for future research on Arabic-speaking ELL university students in the UAE. Currently, only a handful of studies have been done in this specific area, even for ELL university students taken as a whole (Gerber et al., 2005; B Yushau et al., 2005; Balarabe Yushau, 2009; Zepp, 1981). Most of the literature pertains to elementary, middle, and secondary school ELL's in western countries whose primary language (Spanish) is relatively similar to English in terms of orthography and linguistic family. Nonetheless, these are good references and starting points for the study of challenges Emirati university students face 
in this environment. As Moschkovich (2010) suggests, using these studies has its benefits and is applicable as long as the differences between the populations are carefully explained and noted.

This study is intended to provide the background for an action research agenda that practitioners can utilize. It will also be useful for researchers in the field and teachers who work in institutions of higher education that have adopted English as a second language. It is surprising that despite the tremendous amount of time spent and work undertaken by scholars in studying the relationship of language and mathematics learning (Aiken, 1971, 1972; Conference on Psychological et al., 1960; Monroe et al., 1931), very few research-backed solutions have been documented. Hence, it is important to look at these issues more closely and outline a plan to produce and test strategies that will help students learn mathematics more effectively.

\section{Literature Review}

Evidence clearly points to the fact that language issues affect mathematics learning and assessment performance (Abedi et al., 2001; Beal et al., 2010; Bernardo, 2002; Kazima, 2007; Lager, 2006; Stathopoulou et al., 2007). Most studies can be categorized into two major areas: the first concerns studies among immigrants to other countries such as the United States, the United Kingdom, and Australia, whose first language is not that of their new countries. The second concerns students in countries that have adopted English as their primary academic language, where all students are learning English and other subjects at the same time. The two cases are different because for the first, the ELL's are placed in a new environment, where they are immersed in English-rich experiences, they are the minority, and the teachers are mostly native English speakers. In the second category, neither instructor nor student is a native speaker. The teachers and students do not have the rich English-language experiences outside the classroom as ELL's in predominantly English-speaking countries do and they are not the minority. The UAE is a hybrid of the two because the country adopted English as the primary academic language, but most of the instructors in universities are expatriate workers who teach in English and do not speak Arabic. As such, teachers may not always be aware of the problems the students are facing and students have fewer experiences immersing themselves in the language outside the classroom.

\section{Lexical, Syntactic, and Semantic Issues}

In trying to improve the performance of ELL's, the area that teachers would usually consider first is vocabulary and vocabulary building among students. In a study among $6^{\text {th }}$ and $8^{\text {th }}$ grade Latinos, it was found that both ELL and non-ELL's had confusion with vocabulary, but the accumulation of unknown words affected ELL students' performance to a higher degree (Lager, 2006). The author observed that although new mathematical words were explained in the classroom, new technical vocabulary was not always taught. Martiniello (2008) argues that 90 to $95 \%$ of the text must be known to the student for it to be understandable (as cited in Carver, 1994; Nagy, et al., 2000). High-frequency words are likely to be known by the students (Chall et al., 1995; Hildreth, 1948; McKeown et al., 1985); but there is a caveat. Because the students are not from the United States where these studies were conducted, the corpus of words that were considered "high-frequency" for them will not be the same for Emiratis. Efforts are now being made to produce a list specific to the UAE, and in the future, this will be a valuable resource for ELL instructors in this region (Randall et al., 2009). In addition, ABP has a program called the ZU Vocabulary Lab that aims to expand the students' vocabulary base. They are taught the first 3,000 words from the British National Corpus, but Atkinson et al. (2011) show evidence that this program is not effective in terms of acquisition. For now, care must be taken in labeling a word "high-frequency," in other words a lexical item familiar to students, because current data is still incomplete. This also covers polysemous words which Martiniello (2008) argues influence the difficulty of the text. For example, it would be rash for a teacher to assume that domain - either used in the context of domain of a function or synonymous to territory - would be confusing because the students might not even have learned its non-mathematical definition.

There is another problem with vocabulary that presents itself uniquely in this situation. Most of the teachers in the UAE are expatriates, not only from one country, but also from other countries where English is spoken as a first, second or other language. Their English differs from the English learned by 
Emirati students. As argued by Kazima (2007), the teachers' and students' words might be the same, but the way they use them might be slightly or radically different. In her study among Malawian students, she found that they interpreted the words "likely," "unlikely," and "certain" in different ways. They had a tendency to equate "likely" with "certainty" and "unlikely as impossible." On the other hand, "certain" was interpreted as having "some chance." Austin et al. (1979) explain that quantifiers (in a mathematical sense), words such as "always," "everywhere," "sometimes," "in general," "for all," and "with few exceptions," can be particularly confusing. In another study among ELL Papua New Guinean elementary students, it was found that they were 2 years behind in their understanding of the words "more" and "less" as their first-language contemporaries (Jones, 1982). In general, vocabulary issues arise because of two reasons: because they have not learned the words yet, whether mathematical or non-mathematical jargons or words, or because they have acquired a different definition of the word.

Language challenges go beyond the level of the word or phrase (Schleppegrell, 2010). In fact, words used in problems do not necessarily reflect their structural complexity, but still affect comprehension and translation of the problem statement (as cited in Nesher and Teubal, 1975; Hudson, 1983) (Adetula, 1990). Studies have been conducted analyzing how phrases and sentences are constructed, which syntactic features are difficult for ELL's, and which sentence surface characteristics affect comprehension. For example, Austin et al. (1979) list: complicated sentence constructions, long sentences, not using words and phrases frequently, using a lot of new words at a time, using the passive voice, and conditional clauses (if _ _ then _ _). In addition, Abedi et al. (2001) outlined similar linguistic features such as unfamiliarity or frequency of non-mathematical vocabulary, the use of passive verb forms as opposed to the active form, the length of nominals (for instance "the distribution of the averages of the grades of the students" in contrast to "the distribution"), the presence of conditional clauses, the presence of relative clauses, complex question phrases, and abstract or impersonal presentations.

Martiniello (2008) added other features to the list and included: word length, number of prepositional phrases and participial modifiers, and the presence of relative, subordinate, complement, adverbial, or conditional clauses. In her paper, she carefully explains the issues in several mathematics word problems and analyzes the structures that make them complicated. Another researcher focused on the verb "to be" and the relational clauses they form, which can be attributive (such as "A square is ..."), or identifying (" $X$ is equal to $Y$. "), and which may raise issues for ELL's because these structures are constructed differently in other languages (Veel, 1999). Arabic is a notable example because the verb to be is not generally used with the present tense meaning (C. A. Ferguson, 1968).

Although there are many hypothesized linguistic characteristics that affect performance, there is a lack of data showing which particular structures have the greatest negative impact. One study by Shaftel et al. (2006) analyzed data from the United States encompassing 594 unmodified test items from a major state assessment for students in three grade levels. They found that for $4^{\text {th }}$ grade ELL's and students with disabilities, ambiguous or polysemous words, mathematics vocabulary, pronouns, prepositions, and complex verbs (verbs with 3 or more words such as "had been going" and "would have eaten") had statistically significant negative impacts. The items appearing first on the list had the greatest impact. For $7^{\text {th }}$ graders, comparative terms and mathematics vocabulary had a negative effect. This was probably because content with a lot of comparative terms was tested at this level. Finally, for $10^{\text {th }}$ graders, problems arouse due to mathematics vocabulary, followed by complex verbs, which interestingly had a slightly positive effect, and then comparative phrases. The researchers also concluded that $4^{\text {th }}$ graders were more influenced by language probably because of their less sophisticated verbal skills in comparison to $10^{\text {th }}$ graders. For the linguistically more advanced $10^{\text {th }}$ graders, only the difficult mathematics vocabulary influenced their performance. This is important to note because English-proficient Emiratis may have a different experience compared to those with a lower level of language proficiency. In contrast to this finding, a study among 17,000 $3^{\text {rd }}$ grade students did not show evidence for the impact of vocabulary, passive voice constructions, subordinate clauses, and participial modifiers, but did show that item length and the type of response in the assessment had an impact (Lord et al., 2000).

All of the lexical and syntactic issues potentially affect the readability of mathematical text and problems. Many have proposed readability formulas to quantify this attribute, many using factors 
such as number of dependent clauses, number of personal references, number of prepositional phrases, number of words, and number of low frequency words (Gunning, 2003). In the same paper, the author describes one of the most validated indicators, the new Dale-Chall Readability Formula (Chall et al., 1995), which uses average sentence length and the number of words not found on the Revised Dale List. This is a compilation of 3,000 words known by $80 \%$ of fourth graders in the United States. However, the formula was not designed to be used for mathematical text. In fact, a study demonstrated that the readability levels of problems presented to elementary students did not affect their ability to solve them, nor did it interact with grade, problem type (addition, subtraction, multiplication, division, or mixed), and adjustment method (language simplified by changing vocabulary, or number of vocabulary, number of sentences, and length) (Paul et al., 1986).

Another issue researchers have investigated is the difference between Ordinary English (OE) and Mathematical English (ME) (Kane, 1967). The jargon unique to mathematics, the polysemous words that are also present in $\mathrm{OE}$, the syntactical structures, together with signs and symbols, form the language of ME. It evolved from OE because it allowed more flexibility to convey mathematical meaning - to describe matters of degree and quantitative variation in natural phenomenon (Lemke, 2003). This is why it is not practical to think that students can learn ME by learning OE (Schleppegrell, 2010). Moreover, ME is not part of the syllabus of the Academic Bridge Program at $\mathrm{ZU}$ and as students are only exposed to ME in the mathematics classroom, the transition can be very difficult. ELL's may be disadvantaged because they are learning OE and ME at the same time and the differences between the two might not be immediately apparent, nor deliberately taught. On the other hand, it is also possible that there might be less confusion for Emiratis because they may not have learned the overlapping vocabulary and structures in $\mathrm{OE}$ and ME. For example, the word "unique" in ME means "only one exists." Non-ELL's may be confused because it can be used colloquially to mean "not as common," for example, "Her shirt is unique." If Emiratis have not learned to use the word in everyday situations, there will not be any confusion. At this point, we do not know which concepts cause difficulty for ZU students because of the OE/ ME overlap. However, there is reason to believe that the areas that caused confusion for the non-ELL participants from the above studies would also cause confusion for learners at ZU.

Morgan (1996) notes that descriptions of mathematical language have tended to focus on vocabulary, symbolism, and some grammatical structures not commonly found in everyday language. To this, O'Halloran (1998) responds by arguing that mathematics is multi-semiotic. Meaning is conveyed through symbols, visual displays, and language and therefore should be analyzed with all of these elements. She focuses on what she thinks was problematic, the interaction between symbols and language. She argues that difficulties arise because of the degree of clausal rankshift that occurs, which makes text very dense and therefore inaccessible. An example she gives is "s $s(t)=-16 t^{2}+80 t \therefore$ $s(t)=-16 t(t-5), "$ which is formed with terms $(16, t, 80,+)$, expressions $\left(-16 t^{2},-16 t^{2}+80 t\right)$, clauses $(s(t)=-$ $16 t^{2}+80 t$ ), and clause complexes (the whole example). Ideas are embedded in big ideas, which are embedded in even bigger ideas in the same way that Russian Matryoshka dolls are placed one inside the other. She also comments about the long chains of reasoning that provide little or no indication of the results, definitions, axioms, properties, or laws that have been used. The logical relations between lines of mathematical argumentation often remain implicit (O'Halloran, 1998). These force students to make semantic leaps (as cited in Halliday, 1993), which happen frequently during mathematical discourse. This affects students regardless of their language skills, particularly ELL's.

\section{Contextualizing Mathematics Text}

Fasheh (1982) argued for a more culturally aligned mathematics in the classroom when he asked, "If culture determines the way we see a camel, ... may it not also determine the way we think, the way we prove things, the meaning of contradiction, and the logic we use?!" The learning and teaching of mathematics is not universal and culture-free, but often, expatriate teachers in the UAE are not fully conscious of this. Nevertheless, cultural differences do not always prevent understanding from taking place. Emirati students, who use social networks, smart phones, and buy the latest fashions share many traits with typical university students elsewhere. Nonetheless, it is still worth investigating this area because some evidence from previous research points to performance increases with culturally based lessons. There is a call for teachers to provide culturally relevant mathematics (Gutstein et al., 1997), and be 
social anthropologists as well, to determine outside-school mathematical knowledge that is considered legitimate inside the classroom, and also to know about the learners' cultures that can help them in classroom decision-making (Bishop, 1994; Gerdes, 1988). It is not without its challenges, but it is possible to contextualize mathematics lessons just as Mãori teachers have done in New Zealand (Meaney et al., 2013).

Different societies codify their environment in different ways. This is what Stathopoulou et al. (2007) discovered when Romany students in Greece could not understand " $3+2=$ " but were able to when expressed as a verbal problem. They also found that students had difficulty with the instruction, "Mark the shape of the same size" because of their lack of terms for shape, size, and area. In addition, it was also found that when indigenous teachers in Brazil produced their own text for their classes, they utilized a lot of narratives and drawings, not necessarily following the linear and predominantly textual style of western textbooks (Mendes, 2007). Perhaps, mathematical strategies used outside school can be discovered and applied in the classroom to increase comprehension, similar to counting strategies found among child street vendors by researchers in Brazil (Brenner, 1998a; Carraher et al., 1985; Saxe, 1988). For Arab speakers, confusion might arise with writing direction, for example, 45 written as 54, or $(-\infty,-3)$ written as $(-3,-\infty))$, and confusing "." with 0 , and "o" with 5 (Balarabe Yushau, 2009). This might be a case of hypercorrection (Al-Shormani et al., 2012). Since the writing direction for numbers and text in Arabic are not the same, students probably assume that the same is true in English and even once they discovered that it is not, habitually confusion and therefore errors are likely to arise.

Some studies point to the idea that mathematics activities, and correspondingly the instructions that come with them, may be understood in different ways. In a study between Mexican and British 16 to 18 year olds, it was found that the Mexicans preferred strategies that produce precise and algebraic answers, while the British were comfortable with estimation (Molyneux-Hodgson et al., 1999). In addition, it was discovered that the way they used spreadsheets to mathematically model a Chemistry question was different based on their preferences for accuracy or approximation. Of course, the answers sometimes varied as well. It can be hypothesized that matching these preferences to the activities influences comprehension and also performance. In another study, it was found that using curricula based on traditional Yup'ik Native Alaskan customs produced significant and robust performance improvements among $2^{\text {nd }}$ graders (Kisker et al., 2012). However, it is unknown whether the effects were due to better text comprehension or other intertwined factors.

Sometimes, the real world examples that are used in class to bridge classroom mathematics to the world outside disadvantages the students because of how they are used by instructors and the textbook (Brenner, 1998b). This was seen in a study among elementary Hawaiian students when the way the children were using money was different to how they were discussing it in class. At ZU, there are classes that teach the time value of money despite the fact that charging interest is not permissible in Islam, which means there is a mismatch between classroom lessons and societal practices. In addition, situating learning, meaning using local scenarios in mathematical text, might have a negative impact because students lose their focus on the subject (Kaminski et al., 2008). It is something that has to be balanced in the classroom setting. As (Anderson et al., 1996) point out, cognition is partly context-dependent, and is also partly context-independent. Furthermore, they argue that both abstract instruction and training in a social context help. For teachers and researchers in the UAE, the goal is to find this right amount of contextualizing.

\section{Teaching Strategies and Accommodations}

Several studies on the effectiveness of strategies to help mitigate the effect of language proficiency to mathematics performance have been conducted; however, a common limitation is that these strategies were implemented in summative assessments such as state exams in the United States. Because these strategies and accommodations have not been fully studied while being used in the classroom, their potential to help ELL's is not really known. For instance, it is possible that extra time for answering questions may not help students when they take a state exam, which is taken only after weeks, months, or even years of mathematics study. However, this extra time may be very beneficial when used by the teachers during the school year while students learn the content. In practice, it is 
easier to study these after-the-fact interventions, but it would be more fruitful to understand the effects of interventions made in the classroom.

One of the most commonly studied accommodations is language simplification. It is known that English language proficiency affects performance in content-based assessments (Abedi, 2003). Based on previous findings on language characteristics that affect test results, this is intuitively where most research begins. Rewording verbal problems so that the semantic relations are made more explicit, allows children (non-ELL's) to construct more appropriate mental representations for addition, subtraction, and comparison problems (De Corte et al., 1985). The same results were found for $2^{\text {nd }}$ grade Filipinos students, who were all ELL's (Bernardo, 1999).

In contrast to the above, other studies show either no evidence that language simplification has an impact or some evidence for low to moderate effects - if done in summative assessments, after-thefact. If interventions only occur for one final exam and not while the students are learning in the classroom, then obviously, their effects would not be efficacious. A meta-analysis of 11 studies, with a total of 23,999 participants, 6,554 of which were ELL's, and six by Abedi, found that the effects of simplified English was not statistically significant (Kieffer et al., 2009). The same findings are reflected in Shaftel et al. (2003). On the other hand, the Kieffer et al. study highlights using English language dictionaries and glossaries, the only accommodation they found that had an impact. They also found that extra test time did not yield improved test scores for any students. In contrast, Abedi et al. (2000) argues that linguistic modification is the only accommodation that narrows the gap between ELL's and non-ELL's despite the fact that their data show that the difference was narrowed only because of the poorer performance of non-ELL's on the modified questions. Abedi's other study reflects the same finding by Kieffer et al. that other accommodations such as extra time and dictionaries impacted performance (Abedi et al., 2004).

Because studies on accommodations are rare in this region, it is worth mentioning B Yushau et al. (2005) and their work with Saudi university students. They tested glossary use in class for the first two chapters of their textbook, but the results were inconclusive because they did not make a comparison between the performances of students using this glossary and a control group.

Aside from textual improvements, using more visuals and diagrams with the text may also be promising. This goes back to the Mendes (2007) study that found indigenous Xingu teachers from Brazil utilizing narratives combined with culturally tied drawings. The meaning in writing is connected to the pictures and does not only serve as visual support; verbal, numerical, and drawn features were all incorporated in mathematical problems. In a small study among $2^{\text {nd }}$ grade Filipino ELL students, researchers observed that it was necessary for some children to see pictorial, symbolic, or verbal representations before they were able to conceptualize the more complex additive structures (Verzosa et al., 2013). There is some evidence, although not specific to ELL's, pointing to visualization as beneficial in problem solving (Abdullah et al., 2012; Arcavi, 2003; Booth et al., 1999; Presmeg, 1991; Zahner et al., 2010).

In addition, Edens et al. (2008) found that students who used schematic visual representations, as opposed to pictorial, were more successful problem solvers. A visual is schematic if it contains information that supports problem solving, while pictorial visuals include expressive and extraneous elements. However, it can be argued that providing Emirati students both types of visuals would have benefits. Using schematics may make information presentation more efficient (Larkin et al., 1987), and pictures would clarify the context of the problem and help circumvent lexical and semantic difficulties. It might be useful to look into the practices of countries that have adopted English as a second language and that have very successful mathematics programs. One such country is Singapore, which uses textbooks that have low text density and more visual elements (Erbaş et al., 2012).

\section{Classroom Anecdotes}

The aim of this section is to describe examples of scenarios showing the difficulties Zayed University students face in comprehending mathematical text. Word problems previously presented in class were also analyzed to show how findings about linguistic modification might be applied to the 
current materials used in class. These cases were not collected using a structured data gathering methodology. These were simply regular classroom situations that were observed throughout one semester. Most issues that affect ZU students are still not fully understood at this point; future research is necessary to understand these problems and to find ways to solve them.

\section{Scenarios}

A simple confusion students have is the difference between a period and a comma for numbers. For example, one thousand dirhams and 50 fils might be written "1.000,50 dirhams" instead of "1,500.50 dirhams." Although not erroneous, it causes errors especially with calculator use when multiple standards are followed. A related issue about conventions in writing is Arabic's right-to-left writing style. Some get confused when writing or saying numbers. For example, a student was asked to calculate the slope with points $(175,25)$ and $(140,45)$. The answer provided was - 0.75 instead of 0.57. Although the coordinates were determined correctly from the problem, with $\mathrm{x}$ and $\mathrm{y}$ not interchanged, the answer was still incorrectly written probably because of haste. This was also observed by B Yushau et al. (2005) among Saudi university students. This error also appeared when a student used a previously found function to solve another question. The student wrote the function, profit $(x)=1.8 x-120$, but with the next question, "How many $x \ldots$ make a profit of AED 220 million," the student wrote $220=120-1.8 x$, reading the right side of the equation from right to left.

Mistakes related to vocabulary comprehension are more frequently observed in class. In a Business Mathematics class, non-mathematical technical words might confuse students. For example, words such as "invested" or "investment" at the beginning of the word problem, like "The company initially invested $x \times x$ million..." made the question incomprehensible despite students knowing the appropriate formula for this question. In another case, a student did not know the word "initially" and could not determine whether the data was for the present or future value of money (lesson on simple interest). Another very common case is with the word "double." In this phrase, "The future value was double the amount of the present value," the present value was not determined because many students did not know the definition of this word.

Sometimes, it is not just the vocabulary but also the position of the word in relation to the number it is referring to. This question was very problematic for many students:

$$
\text { "A company invested ... and received a AED } 150 \text { interest... Calculate the interest rate." }
$$

Because the word interest was on the next line, students did not understand that AED 150 was the interest amount. This mistake was not very obvious and was only discovered when students asked about this question.

A more surprising problem that remained unnoticed for a long time was discovered when students asked what the word "dice" meant in a Statistics final examination. Dice are probably the most used objects for explaining probability concepts not only in this class, but also in all undergraduate introductory statistics textbooks. The fact that they did not know what this word meant after a whole semester of problem solving means that many concepts were not understood at all.

\section{Problematic Linguistic Features on Word Problems}

Two word problems, one from Business Modeling and the other from Business Statistics, are presented to demonstrate problematic linguistic structures. They were produced internally by mathematics faculty and were not edited in any way by language experts. These were analyzed based on linguistic features identified and listed by Abedi et al. (2001) and Martiniello (2008). The following linguistic modifications simplify text and might lead to greater comprehension. Such modifications include: altering unfamiliar lexical items; turning passive voice as opposed to active; shortening long noun phrases; simplifying complex clauses; and removing abstract or impersonal phrases. In addition, two main problem areas were identified, the first concerning lexical features while the second deals with features of a syntactic nature: Lexical - low frequency items, abstractions, polysemy, culture specific or idiomatic non-math vocabulary terms, and Syntactic - mean sentence 
length, item length in words, length of noun phrase, the number of prepositional phrases and participial modifiers, and complex sentences.
Example 1: calculated).
a. What is the total cost of the tax preparation service?
b. How much extra delivery charge was paid?
c. In effect, what is the percent change from the original price to the final price paid?

Tax preparation services cost AED 360. The company has a 10\% off promotion for loyal customers but an extra delivery charge of $12 \%$ is added to the final price (after promotion is

The item with its three subsequent questions at first might appear to be relatively straightforward and should not to cause difficulties either from a linguistic or an operational point of view. However, a number of issues arise on closer inspection. The first concerns the topic and its context. Generally, the UAE government does not collect personal taxes; therefore students will not be familiar with the concept of tax preparation services. Is somebody being charged a service preparation tax amounting to AED 360 and indeed, did this occur in the past with a number of different services or is this always the case for one service? The main verb cost is irregular and does not contain this information. Lower level students might be initially confused, which is likely to increase text anxiety leading to raised levels of what might be termed textual insecurity (Zeidner, 1998, p. 275).

\section{The company has a $10 \%$ off promotion for loyal customers...}

For the second sentence, the subject, "The company" is also confusing. The reader must now make the assumption that this company is the one that is responsible for the tax preparation service. While this may be patent to a higher-level speaker, given the previous discussion on the possible cultural knowledge gap, here it might not be so obvious. To continue, this company "has a 10\% off promotion...." The noun phrase is likely to cause some readers difficulty, particularly the word "off." It might not be immediately apparent that here it signifies reduction, of course crucial to the solution, rather than its other multifarious meanings. Comprehension is further threatened when we introduce for whom this promotion is for - the loyal customer. While we would expect "customer" to cause no difficulty, the lower frequency "loyal" might.

At this point it might make sense to end that sentence and begin anew. Instead, "but" is added to form a compound sentence, obviously lengthening the text thereby creating further potential problems.

Given the ubiquity of fast food deliveries in the UAE, the concept of a delivery charge should not be troublesome, although the inclusion of the passive "is added" should be noted. Finally the parenthetical ending is also crucial to the answer. Will students be able to refer back to the beginning of the text in order to ascertain the key nature of this information; after all, it comes in brackets - a sign of just something extra, and therefore of less importance? Only a detailed analysis of students' answers in tandem with "think aloud" interviews will reveal to what extent, if at all comprehension was disrupted, which, if this were the case, would use valuable test time at best, and at worst result in the wrong solution being given.

The three questions come next. The first does not appear problematic, but the second contains the past passive, "was paid." The third question begins with a redundant expression and ends with a potentially complex 4-word noun phrase, "the final price paid." The reduced relative clause here simply ending with a participle might cause difficulty. A further cultural issue arises, in that "final price" and "best price" are often heard in the local region. A commonality in English locally used in the UAE, this would mean that no further bargaining is possible, which of course is not what is meant here.

Based on this analysis, a modified version of the above task might read as follows: 
A company that helps people pay tax correctly charges AED 360 for the service. Customers who use the service regularly get a $10 \%$ reduction. However, the company charges $12 \%$ for delivery after calculating the reduction.

a. What is the total cost of the tax service?

b. How much does the delivery cost?

c. What is the percent change from the starting price to the final price?

In the modified task, a number of changes have been made: tense ambiguity has been eliminated utilizing the regular verb "charge;" the function of the company is clarified with a defining relative clause "that helps people pay tax correctly;" passives (is added, was paid) have been removed in favor of active structures; question forms have been simplified, for example "How much does the delivery cost?" as opposed to "How much extra delivery charge was paid?"; redundant vocabulary has also been removed, for example "extra delivery charge" and "in effect"; instead of using the complex noun phrase, "a 10\% off promotion," the modified version reads "customers who... get a 10\% reduction"; and finally, lower frequency vocabulary has been reworded, for example "loyal" to "customers who use the service regularly." Without this modification, the original sentence might be read as "for customers."

The next example was for students at a considerably higher level. They must have a greater ability in mathematics, and it can be assumed therefore that they should have a correspondingly higher level in English. Many students reported the taxing nature of the problem, and it seems apparent this was caused by difficulty in comprehending the language rather than being due to gaps in mathematical knowledge.

\section{Example 2:}

A restaurant owner claims that the mean of his cash sales is "no more than $\$ 3,500$, " but an Internal Revenue Service agent believes the trader is untruthful. Observing a sample of 38 cash customers, the agent finds the mean purchase to be $\$ 3,856$, with a standard deviation of $\$ 234$. Assuming the population is approximately normally distributed, and using the 0.05 level of significance, is the agent's suspicion confirmed?

The task has three sentences and has a word count of 68 . There seems little point in demonstrating complexity through a readability test. Also, at this stage of the students' mathematical career, can it be assumed that they now know that many countries have a tax system and that organizations such as the IRS exist? Again, if such concepts are not part of the culture, then naturally comprehension must suffer. Irrespective of that, syntactic complexity in conjunction with a number of potentially awkward lexical items may cause difficulty. At this stage of learning, can we assume that "population" indicates the entire set of $x$ and not the number of people living in a country? Also it is key to the task that students are aware that the restaurant owner might not always tell the truth, meaning he could be a liar. To say that he is "untruthful" might obfuscate critical meaning.

Syntactically, the second sentence begins with the participle "Observing" and the literal agent is not introduced until the second clause. This type of clausal reversal cannot but hinder the student with a lower than average level of English irrespective of their operational mathematics knowledge. The third sentence also begins with a present participle and is further complicated by the double adverb "approximately normally," which is difficult to modify due to the nature of real world versus hypothetical normal distribution. Clearly "normal distribution" cannot be re-formulated in any other way linguistically; nevertheless, the ME meaning of "normal" might need reinforcing, that is there is no such concept as abnormal distribution, yet other types of mathematical distribution are possible, for example Poisson or Binomial. Finally, the task can only be successfully completed if the rather oblique question at the end is understood, "is the agent's suspicion confirmed?" Surely the inclusion of linguistic obstacles such as this must at the very least be questioned; although one could strongly argue the case for authenticity within any math task at any level, students must be given maximum opportunity to demonstrate their competency in mathematics, not their reading comprehension skills in a second, third or other language.

The second task is modified as follows: 
A restaurant owner says that the mean of his cash sales is $\$ 3,500$ at most. However, a government tax inspector does not believe him. The agent studies 38 cash customers and finds the mean purchase is $\$ 3,856$ with a standard deviation of $\$ 234$. If the population is approximately normally distributed and if we use the 0.05 level of significance, who is correct, the restaurant owner or the agent?

It will be clear that some mathematical terms and collocations cannot be changed or be modified. In the above example, standard deviation, normal distribution, and mean remain. However, vocabulary has been modified, participle clauses removed, simple tenses used, and culture specific items have been altered, for example government tax inspector instead of the Internal Revenue Service. It might be hypothesized that the above modifications will make the text, and therefore, the task, easier to do for students, especially for ELL's. Nevertheless, without think-aloud interviews with the target ELL students, it is not certain that the questions will be more comprehensible.

\section{Conclusion and Future Research Direction}

"The nature of education is such that it would be foolish to expect research work in classroom interaction to lead to hard and fast rules regarding the use of language." (Austin et al., 1979)

Improving the students' educational experiences is a process that includes multiple investigations, project piloting, and implementation, which leads to more research. No single study, nor curriculum or textbook improvement project will result in a panacea for all the struggles the students and teachers face. Perhaps a good philosophy to adopt is that of the Japanese concept of Kaizen, or continuous improvement, implementing small changes frequently that add up and result in large gains.

It is clear that there is a problem of comprehension in mathematics classrooms among ZU students. Despite the incomplete understanding on the issues that Arab or Emirati English Language Learners face, the findings researchers in other countries have discovered can be utilized. For example, there is evidence to suggest that linguistic modification is efficacious. However, its effectiveness is less when only implemented for after-the-fact summative assessments and not in the actual classroom setting. There is great potential in contextualizing the content of mathematical text and there is a call for teachers to understand the culture in which they are immersed. The elements of Emirati traditions and day-to-day life activities are valuable resources in teaching mathematical content. It is also important to investigate solutions beyond language. Mathematics is multi-semiotic, and harnessing visuals - both schematics and pictures, can potentially help students understand the text more without being hindered by lexical or semantic obstacles. These might seem like overwhelming tasks for teachers, but they do not need to be executed all at once. A gradual implementation of accommodation at a pace that is manageable would be more beneficial in the long term.

To advance our knowledge of these language issues in mathematics education, more research is necessary. As this paper shows, interdisciplinary research is required for this endeavor. Cooperation among researchers from different fields would be synergistic and could stimulate research activities in new areas. $\mathrm{ZU}$ is structured so that this is possible and this structure can be harnessed in the future. Although the main interest in this paper is the ZU student body, these research endeavors can also have a major impact in the field of English Language Learning, Mathematics Education, and other content areas internationally particularly the Middle East and North African region. Some topics are proposed below, including sample articles, which could be investigated in the future:

1. A word list specific to ZU students and mathematics - This would be beneficial for improving glossaries used in the classroom and could be taught explicitly to students.

2. Studies on semantic structures that affect comprehension among Emirati students - Using a large randomly selected sample of incoming students, those who are currently in the last semester of ABP, a short interview protocol can be implemented to check which features are problematic. A survey can be administered to verify the findings.

3. Studies on linguistic modifications that have the greatest effect - The effect of specific linguistic changes can be measured using a carefully designed experimental setup. 
4. Investigations on textbook characteristics that affect performance: language, visuals, white space and layout (Dwyer, 1967)

5. Research on characteristics of word problems presentation that increase memory retention (Rohrer, 2009)

6. Studies on classroom practices that increase textual comprehension

7. Studies on the effect of schematics and pictures on problem solving

8. Ethnomathematical studies on Emirati traditions that can be used to teach mathematics (Ismail et al., 2010)

9. Historical research on Islam, and Islamic art and geometry that can be utilized in teaching mathematics (Abas; Setia, 2008); Similar to research on Tamil Kolam designs (Ascher, 2002) and Sona geometry from Angola (Gerdes, 2006)

10. Research on how the history of mathematics can be integrated in the classroom (Abdeljaouad, 2006; Katz et al., 2007)

\section{Acknowledgements}

For our students and colleagues in Abu Dhabi and Dubai and Cindy Stiegelmeyer for reviewing the paper

\section{References}

Abas, Syed Jan. Islamic geometrical patterns for the teaching of mathematics of symmetry. Symmetry in Ethnomathematics, 12(12), 53-65.

Abdeljaouad, Mahdi. (2006). Issues in the History of Mathematics Teaching in Arab Countries. Paedagogica Historica, 42(4/5), 629-664.

Abdullah, Nasarudin, Zakaria, Effandi, \& Halim, Lilia. (2012). The Effect of a Thinking Strategy Approach through Visual Representation on Achievement and Conceptual Understanding in Solving Mathematical Word Problems. Asian Social Science, $8(16)$.

Abedi, Jamal. (2003). Impact of Student Language Background on Content-Based Performance: Analyses of Extant Data. CSE Report.

Abedi, Jamal, \& Hejri, Fereshteh. (2004). Accommodations for students with limited English proficiency in the National Assessment of Educational Progress. Applied Measurement in Education, 17(4), 371-392.

Abedi, Jamal, \& Lord, Carol. (2001). The language factor in mathematics tests. Applied Measurement in Education, 14(3), $219-234$.

Abedi, Jamal, Lord, Carol, Hofstetter, Carolyn, \& Baker, Eva. (2000). Impact of accommodation strategies on English language learners' test performance. Educational Measurement: Issues and Practice, 19(3), 16-26.

Adetula, Lawal O. (1990). Language Factor: Does It Affect Children's Performance on Word Problems? Educational Studies in Mathematics, 21(4), 351-365.

Aiken, Lewis R., Jr. (1971). Verbal Factors and Mathematics Learning: A Review of Research. Journal for Research in Mathematics Education, 2(4), 304-313.

Aiken, Lewis R., Jr. (1972). Language Factors in Learning Mathematics. Review of Educational Research, 42(3), 359-385.

Al-Shormani, Mohammed Qassem, \& Al-Sohbani, Yehia Ahmed. (2012). Semantic Errors Committed by Yemeni University Learners: Classifications and Sources. International Journal of English Linguistics, 2(6), 120-139.

Anderson, John R, Reder, Lynne M, \& Simon, Herbert A. (1996). Situated learning and education. Educational researcher, 25(4), 511.

Arcavi, Abraham. (2003). The Role of Visual Representations in the Learning of Mathematics. Educational Studies in Mathematics, 52(3), 215-241.

Ascher, Marcia. (2002). The Kolam Tradition: A tradition of figure-drawing in southern India expresses mathematical ideas and has attracted the attention of computer science. American Scientist, 90(1), 56-63.

Atkinson, Fairlie, Spring, Jerry, \& Davidson, Peter. (2011). The Impact of Explicitly Teaching Vocabulary on Students' Vocabulary Learning. Retrieved March 4, 2014, from http://marifa.hct.ac.ae/2011/1176

Austin, John Langshaw, \& Howson, Albert Geoffrey. (1979). Language and mathematical education. Educational Studies in Mathematics, 10(2), 161-197.

Barwell, Richard, Barton, Bill, \& Setati, Mamokgethi. (2007). Multilingual issues in mathematics education: introduction. Educational Studies in Mathematics, 64(2), 113-119.

Beal, Carole R, Adams, Niall M, \& Cohen, Paul R. (2010). Reading proficiency and mathematics problem solving by high school English Language Learners. Urban Education, 45(1), 58-74.

Bernardo, Allan BI. (1999). Overcoming obstacles to understanding and solving word problems in mathematics. Educational Psychology, 19(2), 149-163.

Bernardo, Allan BI. (2002). Language and mathematical problem solving among bilinguals. The journal of Psychology, 136(3), 283-297.

Bishop, Alan J. (1994). Cultural Conflicts in Mathematics Education: Developing a Research Agenda. For the learning of mathematics, 14(2), 15-18.

Booth, Rhonda DL, \& Thomas, Michael OJ. (1999). Visualization in mathematics learning: Arithmetic problem-solving and student difficulties. The Journal of Mathematical Behavior, 18(2), 169-190. 
Brenner, Mary E. (1998a). Adding cognition to the formula for culturally relevant instruction in mathematics. Anthropology $\mathcal{E}$ Education Quarterly, 29(2), 214-244.

Brenner, Mary E. (1998b). Meaning and money. Educational Studies in Mathematics, 36(2), 123-155.

Carraher, Terezinha Nunes, Carraher, David William, \& Schliemann, Analucia Dias. (1985). Mathematics in the streets and in schools. British journal of developmental psychology, 3(1), 21-29.

Chall, Jeanne Sternlicht, \& Dale, Edgar. (1995). Manual for Use of the New Dale-Chall Readability Formula: Brookline Books.

Clarkson, Philip. (1983). Types of errors made by Papua New Guinean students. Educational Studies in Mathematics, 14(4), 355367.

Conference on Psychological, Problems, \& Research Methods in Mathematics, Training. $(1960,1960)$. Research problems in mathematics education : reports from a conference on psychological problems and research methods, Washington, D.C.

De Corte, Erik, Verschaffel, Lieven, \& De Win, Luc. (1985). Influence of rewording verbal problems on children's problem representations and solutions. Journal of Educational Psychology, 77(4), 460.

Dwyer, Jr Francis. (1967). Adapting Visual Illustrations for Effective Learning. Harvard Educational Review, 37(2), 250-263.

Edens, Kellah, \& Potter, Ellen. (2008). How students "unpack" the structure of a word problem: Graphic representations and problem solving. School Science and Mathematics, 108(5), 184-196.

Erbaş, Ayhan Kürşat, Alacaci, Cengiz, \& Bulut, Mehmet. (2012). A Comparison of Mathematics Textbooks from Turkey, Singapore, and the United States of America. Educational Sciences: Theory \& Practice, 12(3).

Fasheh, Munir. (1982). Mathematics, Culture, and Authority. For the learning of mathematics, 3(2), 2-8.

Ferguson, Anne M, \& Fairburn, Jo. (1985). Language experience for problem solving in mathematics. Reading Teacher.

Ferguson, Charles A. (1968). Absence of Copula and the Notion of Simplicity: A Study of Normal Speech, Baby Talk, Foreigner Talk and Pidgins. Paper presented at the Conference on Pidginization.

Gerber, Ans, Engelbrecht, Johann, Harding, Ansie, \& Rogan, John. (2005). The influence of second language teaching on undergraduate mathematics performance. Mathematics Education Research Journal, 17(3), 3-21.

Gerdes, Paulus. (1988). On culture, geometrical thinking and mathematics education. Educational Studies in Mathematics, 19(2), 137-162.

Gerdes, Paulus. (2006). Sona geometry from Angola: Mathematics of an African tradition: Polimetrica sas.

Gorgorió, Núria, \& Planas, Núria. (2001). Teaching mathematics in multilingual classrooms. Educational Studies in Mathematics, $47(1), 7-33$.

Gunning, Thomas G. (2003). The role of readability in today's classrooms. Topics in Language Disorders, 23(3), 175-189.

Gutstein, Eric. (2007). Multiple language use and mathematics: Politicizing the discussion. Educational Studies in Mathematics, 64(2), 243-246.

Gutstein, Eric, Lipman, Pauline, Hernandez, Patricia, \& de los Reyes, Rebeca. (1997). Culturally Relevant Mathematics Teaching in a Mexican American Context. Journal for Research in Mathematics Education, 28(6), 709-737.

Halliday, M. (1978). Sociolinguistics Aspects of Mathematical Education Language as social semiotic: The social interpretation of language and meaning (pp. 194-204). London: Edward Arnold Ltd.

Hildreth, Gertrude. (1948). A comparison of the Dale, Dolch and Rinsland word lists. Journal of Educational Psychology, 39(1), 4046.

IELTS. (2014). IELTS | Institutions - IELTS band scores. from https://http://www.ielts.org/institutions/test_format_and_results/ielts_band_scores.aspx

Ismail, Mat Rofa, \& Ismail, Habsah. (2010). Exploring Malay-Islamic Ethnomathematics: Al-Khatib's Combinatoric Theory In 'Alam Al-Hussab And Raudah Al-Hussab. Procedia - Social and Behavioral Sciences, 8(0), 735-744.

Janzen, Joy. (2008). Teaching English language learners in the content areas. Review of Educational Research, 78(4), 1010-1038.

Jones, Peter L. (1982). Learning Mathematics in a Second Language: A Problem with More and Less. Educational Studies in Mathematics, 13(3), 269-287.

Kaminski, Jennifer A., Sloutsky, Vladimir M., \& Heckler, Andrew F. (2008). The Advantage of Abstract Examples in Learning Math. Science, 320(5875), 454-455.

Kane, Robert B. (1967). The readability of mathematical english. Journal of Research in Science Teaching, 5(3), $296-298$.

Katz, Victor J., \& Barton, Bill. (2007). Stages in The History of Algebra With Implications For Teaching. Educational Studies in Mathematics, 66(2), 185-201.

Kazima, Mercy. (2007). Malawian students' meanings for probability vocabulary. Educational Studies in Mathematics, 64(2), 169189.

Kieffer, Michael J, Lesaux, Nonie K, Rivera, Mabel, \& Francis, David J. (2009). Accommodations for English language learners taking large-scale assessments: A meta-analysis on effectiveness and validity. Review of Educational Research, 79(3), 11681201.

Kirk, D., \& al-Istirātìīyah, Markaz al-Imārāt lil-Dirāsāt wa-al-Buh ūth. (2010). The Development of Higher Education in the United Arab Emirates: Emirates Center for Strategic Studies and Research.

Kisker, Ellen Eliason, Lipka, Jerry, Adams, Barbara L, Rickard, Anthony, Andrew-Ihrke, Dora, Yanez, Eva Evelyn, \& Millard, Ann. (2012). The Potential of a Culturally Based Supplemental Mathematics Curriculum to Improve the Mathematics Performance of Alaska Native and Other Students. Journal for Research in Mathematics Education, 43(1), 75-113.

Lager, Carl A. (2006). Types of mathematics-language reading interactions that unnecessarily hinder algebra learning and assessment. Reading Psychology, 27(2-3), 165-204.

Larkin, Jill H, \& Simon, Herbert A. (1987). Why a diagram is (sometimes) worth ten thousand words. Cognitive science, 11(1), 65100.

Lemke, Jay L. (2003). Mathematics in the middle: Measure, picture, gesture, sign, and word. Educational perspectives on mathematics as semiosis: From thinking to interpreting to knowing, 215-234.

Lord, Carol, Abedi, Jamal, \& Poosuthasee, Nida. (2000). Language difficulty and assessment accommodations for English language learners. Dover: Delaware Department of Education. Retrieved February, 1, 2006.

Martiniello, Maria. (2008). Language and the performance of English-language learners in math word problems. Harvard Educational Review, 78(2), 333-368. 
McKeown, Margaret G., Beck, Isabel L., Omanson, Richard C., \& Pople, Martha T. (1985). Some Effects of the Nature and Frequency of Vocabulary Instruction on the Knowledge and Use of Words. Reading Research Quarterly, 20(5), 522-535.

Meaney, Tamsin, Trinick, Tony, \& Fairhall, Uenuku. (2013). One Size does not fit all: Achieving equity in Maori mathematics Classrooms. Journal for Research in Mathematics Education, 44(1), 235-263.

Mendes, Jackeline Rodrigues. (2007). Numeracy and literacy in a bilingual context: Indigenous teachers education in Brazil. Educational Studies in Mathematics, 64(2), 217-230.

Molyneux-Hodgson, Susan, Rojano, Teresa, Sutherland, Rosamund, \& Ursini, Sonia. (1999). Mathematical modelling: The interaction of culture and practice. Educational Studies in Mathematics, 39(1-3), 167-183.

Monroe, Walter Scott, \& Englehart, Max Dissette. (1931). A critical summary of research relating to the teaching of arithmetic. Urbana: University of Illinois.

Morales, Romelia V. (1998). Comprehension and Solution Patterns of Simple Math Word Problems by Mexican-American, Bilingual, Elementary School Students. Paper presented at the Annual Meeting of the National Association for Bilingual Education, Dallas, TX.

Morgan, Candia. (1996). "The Language of Mathematics": Towards a Critical Analysis of Mathematics Texts. For the learning of mathematics, 16(3), 2.

Moschkovich, J. (2010). Language(s) and learning mathematics: Resources, Challenges, and Issues for Research. In J. Moschkovich (Ed.), Language and mathematics education: Multiple perspectives and directions for research (pp. 1-28). Charlotte, NC: Information Age Publishing, Inc.

O'Halloran, Kay L. (1998). Classroom discourse in mathematics: A multisemiotic analysis. Linguistics and Education, 10(3), 359388.

Paul, Douglas J, Nibbelink, William H, \& Hoover, Hiram D. (1986). The effects of adjusting readability on the difficulty of mathematics story problems. Journal for Research in Mathematics Education, 163-171.

Pimm, David. (1987). Speaking mathematically: Communication in mathematics classrooms: Routledge \& Kegan Paul London.

Presmeg, NC. (1991). Classroom aspects which influence use of visual imagery in high school mathematics. Paper presented at the PME CONFERENCE.

Randall, Mick, \& Groom, Nicholas. (2009). The BUiD Arab learner corpus: a resource for studying the acquisition of L2 english spelling. Paper presented at the Proceedings of the Corpus Linguistics Conference (CL), Liverpool, UK.

Ríordáin, Máire Ní, \& O’Donoghue, John. (2009). The relationship between performance on mathematical word problems and language proficiency for students learning through the medium of Irish. Educational Studies in Mathematics, 71(1), $43-64$.

Rohrer, Doug. (2009). The Effects of Spacing and Mixing Practice Problems. Journal for Research in Mathematics Education, 40(1), 4-17.

Saxe, Geoffrey B. (1988). The mathematics of child street vendors. Child Development, 1415-1425.

Schleppegrell, Mary J. (2010). Language in Mathematics Teaching and Learning. In J. Moschkovich (Ed.), Language and mathematics education: Multiple perspectives and directions for research (pp. 73-112). Charlotte, NC: Information Age Publishing, Inc.

Setia, Adi. (2008). Some upstream research programs for Muslim mathematicians: operationalizing Islamic values in the sciences through mathematical creativity. Islam \& science, 6(2), 153.

Shaftel, Julia, Belton-Kocher, Evelyn, Glasnapp, Douglas, \& Poggio, John. (2003). The differential impact of accommodations in statewide assessment: Research summary. 2014, from http://education.umn.edu/NCEO/TopicAreas/Accommodations/Kansas.htm

Shaftel, Julia, Belton-Kocher, Evelyn, Glasnapp, Douglas, \& Poggio, John. (2006). The impact of language characteristics in mathematics test items on the performance of English language learners and students with disabilities. Educational Assessment, 11(2), 105-126.

Solano-Flores, Guillermo. (2010). Function and Form in Research on Language and Mathematics Education. In J. Moschkovich (Ed.), Language and mathematics education: Multiple perspectives and directions for research (pp. 113-149). Charlotte, NC: Information Age Publishing, Inc.

Stathopoulou, Charoula, \& Kalabasis, Fragiskos. (2007). Language and culture in mathematics education: Reflections on observing a Romany class in a Greek school. Educational Studies in Mathematics, 64(2), 231-238.

Veel, Robert. (1999). Language, knowledge and authority in school mathematics. In F. Christie (Ed.), Pedagogy and the shaping of consciousness (pp. 185-216). London and New York: Continuum International Publishing Group, Limited.

Verzosa, Debbie Bautista, \& Mulligan, Joanne. (2013). Learning to solve addition and subtraction word problems in English as an imported language. Educational Studies in Mathematics, 82(2), 223-244.

Vinner, Shlomo. (1997). The Pseudo-Conceptual and the Pseudo-Analytical Thought Processes in Mathematics Learning. Educational Studies in Mathematics, 34(2), 97-129.

Yushau, B, \& Bokhari, M. (2005). Language and mathematics: a mediational approach to bilingual Arabs. International Journal for Mathematics Teaching and Learning,(April).

Yushau, Balarabe. (2009). Mathematics and language: issues among bilingual Arabs in English medium universities. International Journal of Mathematical Education in Science and Technology, 40(7), 915-926.

Zahner, Doris, \& Corter, James E. (2010). The process of probability problem solving: Use of external visual representations. Mathematical Thinking and Learning, 12(2), 177-204.

Zeidner, Moshe. (1998). Test anxiety the state of the art. New York :: Plenum Press.

Zepp, Raymond A. (1981). Relationships between mathematics achievement and various English language proficiencies. Educational Studies in Mathematics, 12(1), 59-70.

ZU. (2008). Zayed University Self-Study Report: Prepared for Middle States Commission on Higher Education UAE: Zayed University.

ZU. (2012a). New Directions for Student Success: ABP-COL Curricular Integration. UAE: Zayed University.

ZU. (2012b). Zayed University Self-Study Report: Prepared for Middle States Commission on Higher Education UAE: Zayed University. 